\title{
Improved Composite Q-Function Approximation and its Application in ASEP of Digital Modulations over Fading Channels
}

\author{
Aleksandar Markovic ${ }^{1}$, Zoran Peric ${ }^{1}$, Stefan Panic ${ }^{2}$, Petar Spalevic ${ }^{3}$, Zoran Todorovic ${ }^{4}$ \\ ${ }^{1}$ University of Nis, Faculty of Electronic Engineering, \\ Aleksandra Medvedeva 14, 18000 Nis, Serbia \\ ${ }^{2}$ University of Pristina, Faculty of Mathematics, \\ Lole Ribara 29, 38220 Kos. Mitrovica, Serbia \\ ${ }^{3}$ University of Pristina, Faculty of Technical Sciences, \\ Knjaza Milosa 7, 38220 Kos. Mitrovica, Serbia \\ ${ }^{4}$ Department of Technical Sciences, Singidunum University, \\ Danijelova 32, 11000 Beograd, Serbia \\ aleksandar.markovic@pr.ac.rs
}

\begin{abstract}
In this paper, capitalizing on Mils ratio for Qfunction approximation, we have presented novel improved composite Q-function approximation. Based on our improved approximation, we have further presented tight approximation for the average symbol error probability (ASEP) expressions of digital modulations over Nakagami-m fading channels. First, comparison to other known Q-function closed-form approximations has been performed, and it has been shown that accuracy improvement has been achieved in the observed range of values. Further, it has been shown that by using proposed approximation, values of average symbol error probability (ASEP) for some applied modulation formats could be efficiently and accurately evaluated when transmission over Nakagami-m fading channels is observed. Also, it has been shown in the paper that by using proposed approximation, observed ASEP measures are bounded more closely, than by using other known Q-function closed-form approximations.
\end{abstract}

Index Terms-Function approximation; Gaussian distribution; modulation; Nakagami distribution.

\section{INTRODUCTION}

Rapid development of various wireless communication system services, has resulted in constant need for providing mathematical models of wireless transmission phenomenons. Wireless propagation is accompanied by various side effects and drawbacks, among which most important one is multipath fading. Various fading models are already known in the literature, however, it was shown that Nakagami- $m$ fading model provides best fits to collected data in indoor and outdoor wireless environments [1]. Similarly, best fit to land-mobile and indoor mobile multipath propagation as well as scintillating ionospheric radio links can be obtained by observing Nakagami- $m$ fading model. Nakagami- $m$

Manuscript received 25 November, 2016; accepted 21 March, 2017.

This research has been partially supported by the funds of Ministry of Education, Science and Technological Development (Project III-44006 and TR 32023). fading model describes multipath scattering with large delaytime spreads, and different clusters of reflected waves, it provides good fits to collected data in indoor and outdoor wireless environments [1]. Being a general fading distribution, Nakagami- $m$ fading model includes (as its singularities) other fading models such are Rayleigh distribution (by setting parameter $m$ value $m=1$ ), and onesided Gaussian distribution $(m=1 / 2)$ [2].

In order to predict of behaviour of wireless communication systems, characterized by a variety of modulation types, detection types and channel models, one first must determine standard performance measures of observed wireless communication system and to observe how these performance measures depend on key system parameters values. Performance measure, which usually quantifies the nature of the wireless communication system behaviour, by quantifying the reliability or integrity of a received signal, is the average symbol error probability (ASEP) [3]. ASEP values are obtained capitalizing on conditional SEP relations, which are conditioned over fading statistics which impairs the communication. Conditional SEPs are functions of the instantaneous state of fading channel, and functional dependency is determined by the type of modulation scheme performed. In order to analytically evaluate ASEP for applied modulation format, expression for conditional SEP (conditioned over fading statistics which impairs the communication) should be averaged over the probability density function (PDF) of the fading channel amplitude. However, in many such cases the averaging integral includes either the Gaussian Q-function, either directly related functions: error function, erf $(x)$, and/or complementary error function erfc $(x)$.

The Gaussian Q-function is special function, defined as non-elementary definite integral, so it cannot be expressed as finite composition of basic functions. This property of Qfunction makes conducting communication systems 
performance analysis, so there arise a need for obtaining accurate and easily tractable closed-form approximation. Various approximations have been proposed in the literature so far [4]-[10].

In this paper we will propose approximation to the Gaussian Q-function, obtained based on the properties of Mils ratio approximation [10] for Q-function, but taking the into account composite properties of minimization MSE (Mean-square error). Comparison to other known Q-function closed-form approximations has been performed, and it has been shown that by using proposed approximation accuracy improvement has been achieved in whole range of values. Also, it has been shown that by using proposed approximation, ASEP values for BPSK and DE-QPSK applied modulation schemes could be efficiently and accurately evaluated when transmission over Nakagami- $m$ fading channels is observed.

\section{AN IMPROVED APPROXIMATION OF THE Q-FUNCTION}

We start with the formal definition of the Gaussian Qfunction $\mathrm{Q}(\mathrm{x})$, defined as [11]-[13] follows

$$
Q(x)=\frac{1}{\sqrt{2 \pi}} \int_{x}^{\infty} e^{-\frac{t^{2}}{2}} d t
$$

In the terms of the complementary error function $\operatorname{erfc}(\mathrm{x})$, $\mathrm{Q}(\mathrm{x})$ can be expressed as

$$
Q(x)=\frac{1}{2} \operatorname{erfc}\left(\frac{x}{\sqrt{2}}\right) .
$$

In [4] Borjesson and Sundberg had proposed approximations to $\mathrm{Q}(x)$ in the form of

$Q_{a-\text { Borjesson-1 }}(x)=\frac{1}{\sqrt{2 \pi}} \frac{1}{(1-a) x+a \sqrt{x^{2}+b}} e^{-\frac{x^{2}}{2}}$,

where $a$ and $b$ are scalar fitting parameters, which can be determined following some numerical optimization procedure in order minimize the integral of the absolute error given by

$$
\begin{aligned}
M S E_{\min }= & \frac{1}{N} \int_{0}^{N}\left(Q(x)-\frac{e^{-\frac{x^{2}}{2}}}{\sqrt{2 \pi}\left[(1-a) x+a \sqrt{x^{2}+b}\right]}\right) d x \approx \\
& \approx \frac{1}{N} \sum_{i=1}^{N}\left(Q\left(x_{i}\right)-Q_{(a, b)}\left(x_{i}\right)\right)^{2},
\end{aligned}
$$

with $N$ being the range of argument values of interest.

Namely, solving (4) is equivalent to finding values for parameters $a$ and $b$ for which $\mathrm{MSE}_{\min }$ obtains value which is as small as possible for the considered interval of $x$.

As shown in [4], the combination of $a=0.339$ and $b=$ 5.510, denoted as $\mathrm{Q}_{a \text {-Borjesson-1 }}(\mathrm{x})$, gives the best approximation for $x>0$, namely:
$Q_{a-\text { Borjesson-1 }}(x)=\frac{1}{\sqrt{2 \pi}} \frac{e^{-\frac{x^{2}}{2}}}{0.661 x+0.339 \sqrt{x^{2}+5.51}}$.

Another widely used approximation for $x \in[0, \infty)$ is $a=1$ and $b=1$ leading to [4]

$$
Q_{a-\text { Borjesson-2 }}(x)=\frac{1}{\sqrt{2 \pi}} \frac{1}{\sqrt{x^{2}+1}} e^{-\frac{x^{2}}{2}} \text {. }
$$

However, it has been shown in [4], that all approximations developed by Borjesson and Sundberg are often not very suitable for algebraic manipulations related to communication system performance analysis.

Karagiannidis et al. in paper [6] have proposed complementary error function approximation in the form of

$$
Q_{a-\text { Karagiannidis }}(x)=\frac{1}{\sqrt{2 \pi}} \frac{\left(1-e^{-A \frac{x}{\sqrt{2}}}\right)}{B x} e^{-\frac{x^{2}}{2}} \text {, }
$$

with parameters $A$ and $B$ chosen in order to minimize the integral of the absolute error in the range of interest, $[0, \mathrm{R}]$, i.e.

$$
\{A, B\}=\underset{\{A, B\}}{\arg \min } \frac{1}{R} \int_{0}^{R}\left|Q_{a-\text { Karagiannidis }}(x)-Q(x)\right| d x,
$$

where $|x|$ denotes absolute value. The optimal values of $A=$ 1.98 , and $B=1.135$, for $R=20$, are found numerically, by minimizing the integral of the absolute error [6].

Taking the same form with [6], a simple upper bound of the Gaussian has been very recently proposed by Jang in [7] as follows

$$
Q_{a-J a n g}(x)=\frac{1}{\sqrt{2 \pi}} \frac{\left(1-e^{-\sqrt{\frac{\pi}{2}} x}\right)}{x} e^{-\frac{x^{2}}{2}} .
$$

Isukapalli approximation expression of Q-function can be expressed as [8]

$Q_{a-\text { Isukapalli }}(x)=\frac{1}{\sqrt{2 \pi}}\left(\sum_{n=1}^{n_{a}} \frac{(-1)^{n+1}(A)^{n}}{B(\sqrt{2})^{n} n !} x^{n-1}\right) e^{-\frac{x^{2}}{2}}$,

where $A$ and $B$ are selected according to [7]; $n_{a}$ is the number of selected terms depending on the desired tightness.

Chiani approximation expression of Q-function can be expressed as [5]

$$
Q_{a-C h i a n i}(x)=\frac{1}{12} e^{-\frac{x^{2}}{2}}+\frac{1}{4} e^{-\frac{2 x^{2}}{3}} .
$$


Shi approximation expression of Q-function can be expressed as [9]

$$
Q_{a-S h i}(x)=\frac{1}{\sqrt{\pi}} \sum_{n=1}^{N} \omega_{n} e^{-\sqrt{2} \zeta_{n} x} e^{-\frac{x^{2}}{2}},
$$

where $\zeta_{n}$ and $\omega_{n}$ are the $n$th root of $N$-order Hermite polynomial and the corresponding weights. As illustrated in [9], this approximation result is sufficiently accurate only with $N=2$, i.e., using only two Gauss points.

Prony approximation of the Q-function in terms of two exponential functions has represented in [14]

$$
Q_{a-\text { Loskot }}(x)=0.208 e^{-0.971 x^{2}}+0.147 e^{-0.525 x^{2}} \text {. }
$$

In [15], a very simple result with only one exponential term was derived. The optimum values of fitting parameters $a$ and $b$ were given in [15] as 1.394 and $8 / 13$

$$
Q_{a-\text { Sofotasios }}(x)=0.49 e^{-\frac{8}{13} x} e^{-\frac{x^{2}}{2}} .
$$

Another similar result has recently proposed in [16], also based on the empirical approach, written as

$$
Q_{a-\text { Benitez }}(x)=e^{a x^{2}+b x+c},
$$

where $a, b$ and $c \in R$ are fitting parameters derived by using numerical method. Optimum values of fitting parameter $(a$, $b, c)$ are provided in [16, Table I], for $x \in[0,20]$.

Dao et al. have proposed approximations for $\mathrm{Q}(x)$ function given as [17]

$$
Q_{a-D a o}(x)=e^{a x^{6}+b x^{5}+c x^{4}+d x^{3}+e x^{2}+f x+g},
$$

where $a=-0.0000009, b=0.0000104, c=0.000686, d=$ $0.015205, e=-0.363992, f=-0.762741$ and $g=-0.694072$.

Polynomial Q-function approximation is given by [18]

$$
\begin{gathered}
Q_{a-\text { Chen }}(x)=1-\sum_{m=0}^{n} \sum_{p=0}^{n} \frac{(-1)^{m+p}\left(\begin{array}{c}
n \\
p
\end{array}\right)}{m !(n-m) !}\left(\frac{n}{12}\right)^{\frac{p}{2}} \times \\
\times\left(\frac{n}{2}-m\right)^{n-p} x^{p} U\left[x-\sqrt{\frac{12}{n}}\left(\frac{n}{2}-m\right)\right]
\end{gathered}
$$

where $|x|<\sqrt{3 n}$ and $U(x)$ is the unit step function defined as $U(x)=1$ if $x>0, U(x)=1 / 2$ if $x=0$ and $U(x)=0$ if $x<0$.

Now, let us define novel composite approximation of $\mathrm{Q}(x)$ function with minimal complexity of two regions bounded by $\mathrm{x}_{1}$

$$
Q_{a-M S E_{\min }}(x)=
$$

$$
=\left\{\begin{array}{ll}
\frac{1}{\sqrt{2 \pi}} \frac{e^{-\frac{x^{2}}{2}}}{\left(1-a_{1}\right) x+a_{1} \sqrt{x^{2}+b_{1}}}, & x \in\left[0, x_{1}\right] \\
\frac{1}{\sqrt{2 \pi}} \frac{e^{-\frac{x^{2}}{2}}}{\left(1-a_{2}\right) x+a_{2} \sqrt{x^{2}+b_{2}}}, & x \geq x_{1},
\end{array},\right.
$$

The idea for constructing such composite function has occurred by observing property that accuracy in approximating the Q-function depends on the fitting parameters, which further strictly, depend on the range of argument. Starting from Mils ratio approximation with respect to minimizing MSE value for the considered intervals of $x$, after providing minimization of MSE for observed ranges of $x \in\left[0, x_{1}\right]$ and $x \in\left[x_{1}, \infty\right]$, corresponding values for parameters, $x_{1}, a_{1}, a_{2}, b_{1}$, and $b_{2}$ of $x_{1}=1, a_{1}=0.3442, b_{1}=5.339 a_{2}=0.31152$ and $b_{2}=6.2076$ are obtained.

$$
=\left\{\begin{array}{cl}
Q_{a-M S E_{\min }}(x)= & \\
\frac{1}{\sqrt{2 \pi}} \frac{e^{-\frac{x^{2}}{2}}}{0.6558 x+0.3442 \sqrt{x^{2}+5.339}}, & x \in[0,1], \\
\frac{1}{\sqrt{2 \pi}} \frac{e^{-\frac{x^{2}}{2}}}{0.6885 x+0.3115 \sqrt{x^{2}+6.2076}}, & x \geq 1,
\end{array}\right.
$$

In Table I and Fig. 1 we have presented comparison between $\mathrm{Q}(x)$ function and its approximations. As one can see it is evident that accuracy improvement has been achieved in whole range of values, by using proposed novel approximation of $\mathrm{Q}(x)$, given by (19).

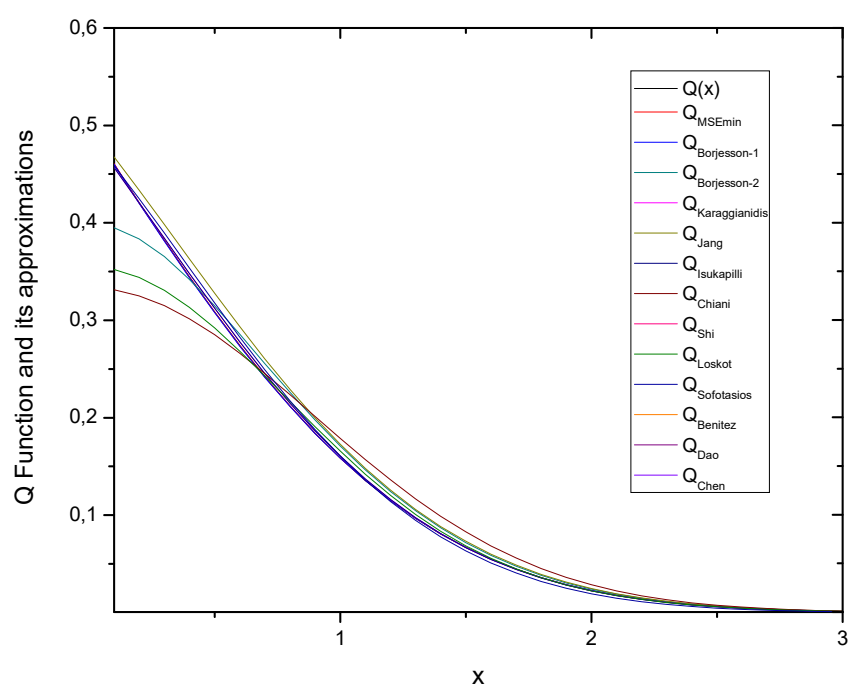

Fig. 1. Comparison between the $\mathrm{Q}(x)$ approximations.

At Fig. 2 is presented absolute relative error calculated for proposed $\mathrm{Q}(x)$ approximation in comparison with other approximations. 
TABLE I. COMPARISON AMONG Q(X) FUNCTION APPROXIMATIONS

\begin{tabular}{|c|c|c|c|c|c|c|c|c|c|c|}
\hline Function & $x=0.1$ & $x=0.3$ & $x=0.5$ & $x=0.7$ & $x=1$ & $x=5$ & $x=10$ & $x=15$ & $\mathbf{x}=\mathbf{2 0}$ & MSE \\
\hline$Q(x)$ & & & & 0.24196 & .15866 & $.8665 \mathrm{e}-07$ & $7.6199 \mathrm{e}-24$ & $3.6710 \mathrm{e}-51$ & $2.7536 \mathrm{e}-89$ & - \\
\hline & 6065 & & & & & 8686e-07 & $7.6220 \mathrm{e}-24$ & $6714 \mathrm{e}-51$ & $.7538 \mathrm{e}-89$ & $3.64 \mathrm{e}-8$ \\
\hline son-1 $(x)$ & 6020 & & 0772 & & & $8715 \mathrm{e}-07$ & $7.6243 \mathrm{e}-24$ & $6719 e-51$ & & $6 e-7$ \\
\hline QBorjesson-2(x) $_{2}$ & 0.39498 & 0.36530 & 0.31490 & 0.25581 & 0.17110 & $2.9157 \mathrm{e}-07$ & $7.6564 \mathrm{e}-24$ & $3.6790 \mathrm{e}-51$ & $2.7570 \mathrm{e}-89$ & $5.5 e-4$ \\
\hline $\mathbf{Q}_{\mathrm{Kar}}$ & 0.45693 & 415 & 1232 & 0.24552 & 62 & $2.6174 \mathrm{e}-07$ & $6.7794 \mathrm{e}-24$ & $3.2486 \mathrm{e}-51$ & $2.4321 \mathrm{e}-89$ & $5.06 \mathrm{e}-6$ \\
\hline & 0.46759 & 0.39842 & 0.32786 & 0.26056 & 287 & $2.9678 \mathrm{e}-07$ & $7.6946 \mathrm{e}-24$ & $3.6871 \mathrm{e}-51$ & $2.7605 \mathrm{e}-89$ & $1.38 \mathrm{e}-4$ \\
\hline $\mathbf{Q}_{\text {Isukapilli }}(\mathbf{x})$ & 0.45693 & 0.38416 & 0.31241 & 0.24595 & 247 & $2.0881 \mathrm{e}-05$ & $2.2070 \mathrm{e}-20$ & $8.8628 \mathrm{e}-47$ & $2.9462 \mathrm{e}-84$ & $6.63 \mathrm{e}-6$ \\
\hline & 0.33126 & 0.31511 & 0.28516 & 0.24556 & 890 & $3.2500 \mathrm{e}-07$ & $1.6073 \mathrm{e}-23$ & $1.1553 \mathrm{e}-50$ & $1.1532 \mathrm{e}-88$ & $2.45 \mathrm{e}-3$ \\
\hline 0 & 0.46017 & 206 & 0.30838 & 0.24154 & 767 & $1.6129 \mathrm{e}-07$ & $9.9883 \mathrm{e}-25$ & $944 \mathrm{e}-53$ & $1.0270 \mathrm{e}-91$ & $1.31 \mathrm{e}-7$ \\
\hline & 0.35222 & 0.33081 & 0.29209 & 0.24291 & 0.16573 & $2.9323 \mathrm{e}-07$ & $2.3273 \mathrm{e}-24$ & $7.3499 \mathrm{e}-53$ & $9.2358 \mathrm{e}-93$ & $1.62 \mathrm{e}-3$ \\
\hline $\mathbf{Q}_{\text {Sofotasios }}(\mathbf{x})$ & 0.45846 & 0.38947 & 0.31789 & 0.24929 & 662 & $3 \mathrm{e}-08$ & $2.0086 \mathrm{e}-25$ & $57 e-54$ & $29 \mathrm{e}-93$ & $2.25 \mathrm{e}-5$ \\
\hline$Q_{\text {Beni }}$ & 0.45995 & 0.38282 & 0.30899 & 0.24185 & 0.15809 & $7.3647 \mathrm{e}-07$ & $4.9426 \mathrm{e}-21$ & $1.5065 \mathrm{e}-43$ & $2.0853 \mathrm{e}-74$ & $1.24 \mathrm{e}-7$ \\
\hline$Q_{\text {Dao }}(x)$ & 0.46002 & 0.38210 & 0.30858 & 0.24200 & 0.15866 & $2.8672 \mathrm{e}-07$ & $9.0226 \mathrm{e}-24$ & $2.0201 \mathrm{e}-46$ & $5.4641 \mathrm{e}-36$ & $2.02 \mathrm{e}-7$ \\
\hline $\mathbf{Q}_{\text {Chen }}(x)$ & 0.46139 & 0.38551 & 0.31352 & 0.24764 & 0.16381 & $1.8652 \mathrm{e}-14$ & $5.6843 \mathrm{e}-14$ & $1.3642 \mathrm{e}-12$ & $3.1832 \mathrm{e}-12$ & $1.07 \mathrm{e}-5$ \\
\hline
\end{tabular}

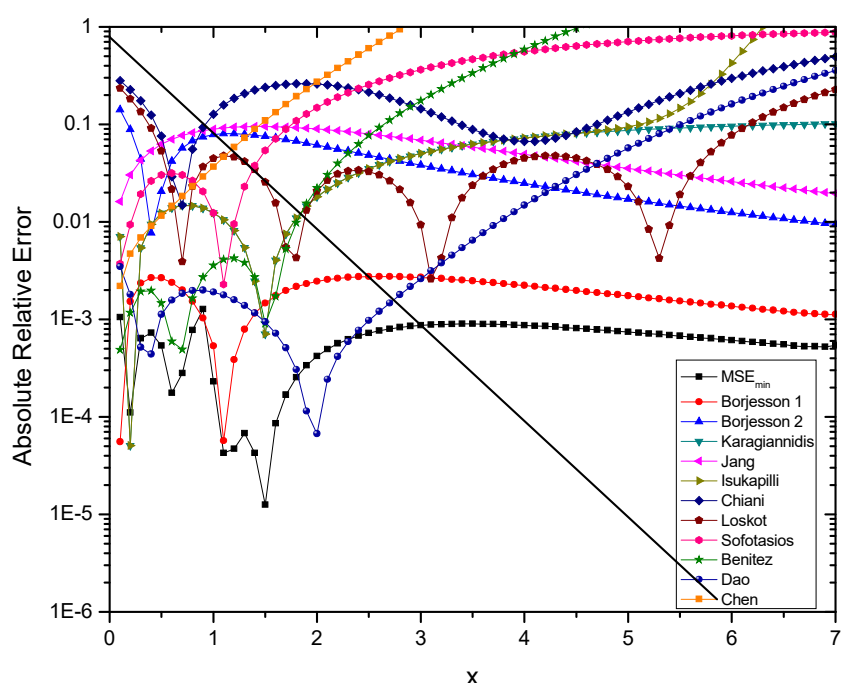

Fig. 2. Absolute relative error for the new approximation of $\mathrm{Q}(\mathrm{x})$ in comparison with other approximations.

As visible from Fig. 2, approximation given with (19) has minimal relative absolute error and it is more accurate fit than other approximations in almost whole range of input arguments.

\section{APPLICATION to THE FADING CHANNELS PERFORMANCE EVALUATION}

Let us now extend our approximation to the case of Average bit error rate (ABER) values evaluation for given modulation scheme over Nakagami-m fading channels. As mentioned, we should average expression for conditional SEP over the probability density function (PDF) of the fading channel amplitude for applied modulation format. With the binary phase-shift keying (BPSK) modulation, we can express the ASEP in the form of

$$
P_{b}=\int_{0}^{\infty} Q\left(\sqrt{\frac{2 E_{b}}{N_{0}}} x\right) f_{x}(x) d x,
$$

where $f \mathrm{x}(x)$ is the PDF of the Nakagami- $m$ fading channel amplitude. $E_{b}$ and $N_{o}$ denote the average bit energy and the one-sided noise power spectral density, respectively.

Nakagami- $m$ distributed random processes, has PDF given in the form of [1]

$$
f_{x}(x)=\frac{2}{\Gamma(m)} \frac{m^{m}}{\Omega^{m}} x^{2 m-1} \exp \left\{-m \frac{x^{2}}{\Omega}\right\},
$$

with $\Omega$ being the average signal power, and $m$ denoting the inverse normalized variance of $x$, describing the fading severity , $\Gamma(m)$ represents the special Gamma function [19], Eq. (8.841.4)].

After substituting our expression for approximation of $\mathrm{Q}(x)$ function, (19) we can efficiently evaluate values for ABER over Nakagami- $m$ fading conditions.

At Fig. 3 is depicted ABER for BPSK over Nakagami-m fading. It can be seen that ABER for BPSK over Nakagami$m$ fading values could be efficiently and accurately evaluated by proposed method for all considered values of parameter $m$. Also can be seen that by using proposed approximation by (19) ABER measures are bounded more closely than by using other known Q-function closed-form approximations in whole range of fading conditions. In order to point out excellent agreement of approximated ABER BPSK values with the exact ones, at Fig. 4 we have presented values for absolute relative error of proposed ABER BPSK approximation for various values of Nakagami- $m$ channel conditions.

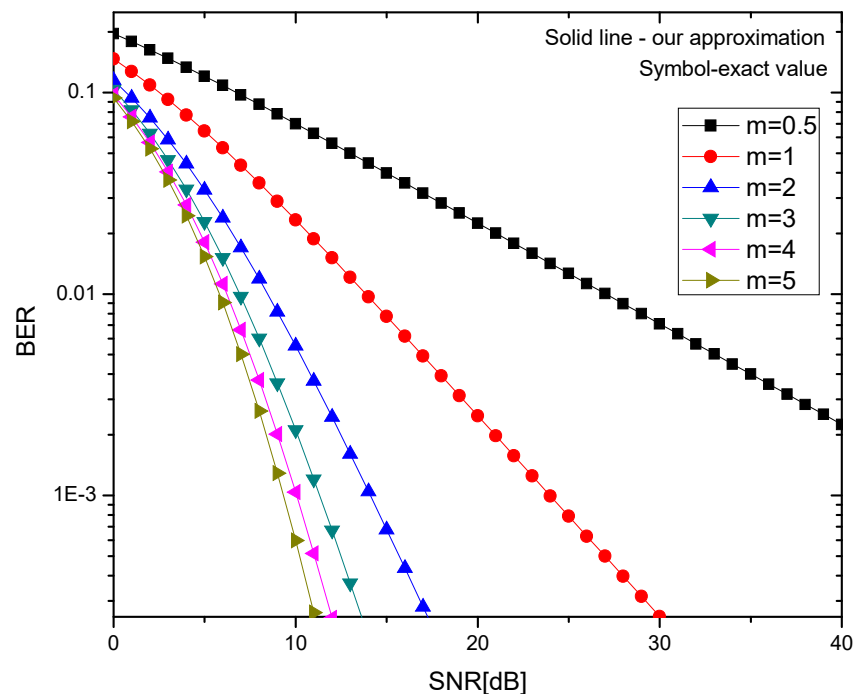

Fig. 3. Average BER for BPSK over Nakagami-m fading. 


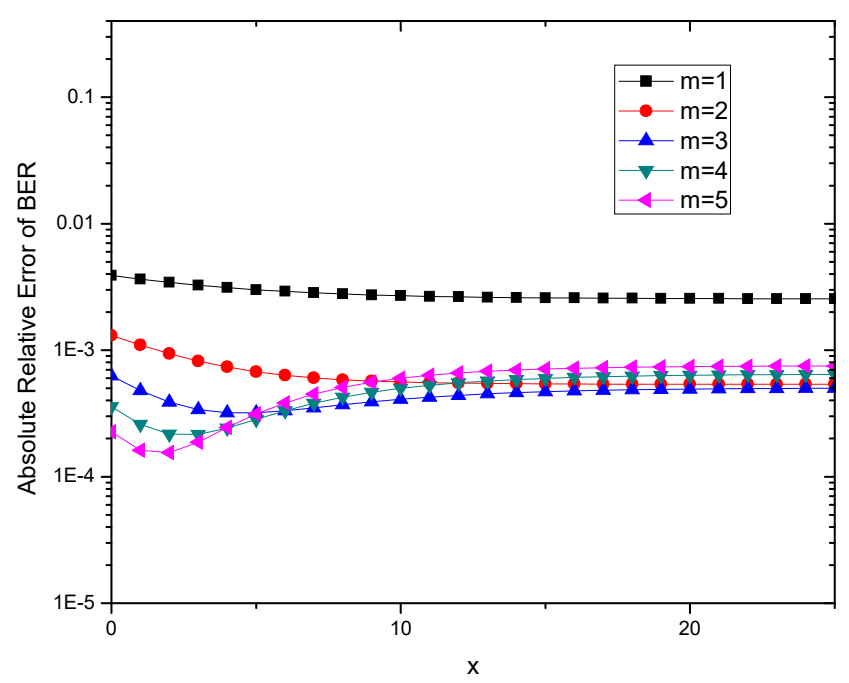

Fig. 4. Absolute relative error of proposed ABER BPSK approximation.

Let us now extend our approximation to the case of ABER over coherent detection of Differentially EncodedQuadrature Phase Shift Keying (DE-QPSK) over Nakagami$m$ fading channels. In [20], it has been shown that the ABER of DE-QPSK can be obtained by averaging

$$
P_{b}=\int_{0}^{\infty} \overline{P_{b}} \times f_{\gamma}(\gamma) d \gamma
$$

where $f \gamma(\gamma)$ is the Nakagami- $m$ PDF of the signal-to-noise ratio (SNR) per symbol, distributed according to

$$
f_{\gamma}(\gamma)=\frac{m^{m}}{\Gamma(m) \bar{\gamma}^{m}} \gamma^{m-1} \exp \left\{-m \frac{\gamma}{\bar{\gamma}}\right\},
$$

with $\bar{\gamma}$ denoting the average SNR value of observed instantaneous process. In the case of (DE-QPSK) error probability has the expression [11]

$$
\overline{P_{b}}=4 Q(\sqrt{\gamma})-8 Q^{2}(\sqrt{\gamma})+8 Q^{3}(\sqrt{\gamma})-4 Q^{4}(\sqrt{\gamma}),
$$

At Fig. 5 is presented ABER for DE-QPSK over Nakagami- $m$ fading.

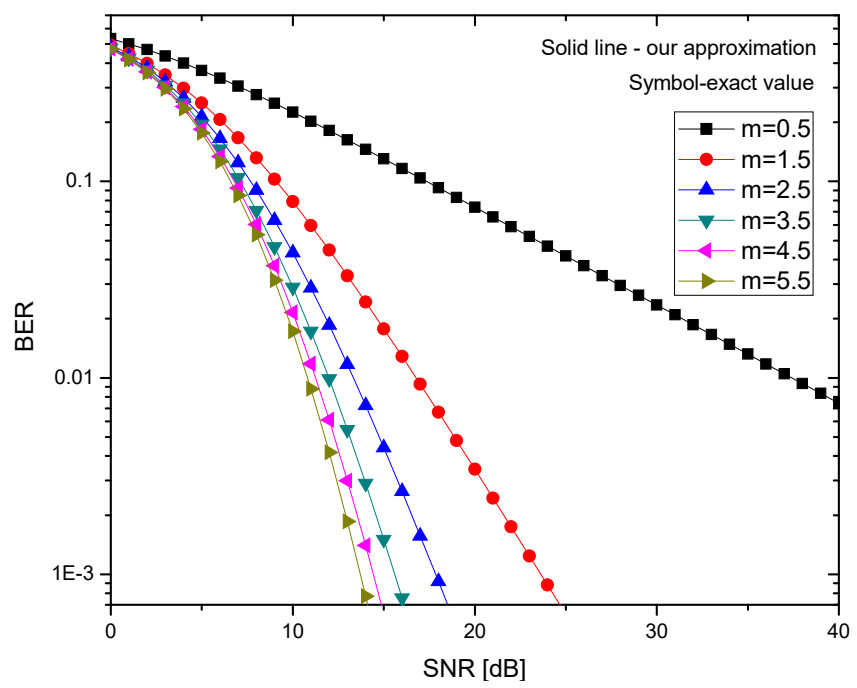

Fig. 5. Average BER for DE-QPSK over Nakagami-m fading.
From Fig. 5, it can be seen that ABER for DE-QPSK over Nakagami- $m$ fading values also could be efficiently and accurately evaluated by proposed method for all considered values of parameter $m$. Also can be seen that by using proposed approximation by (19), ABER measures are bounded more closely than by using other known Q-function closed-form approximations.

At Fig. 6 we have presented values for absolute relative error of proposed ASEP DE-QPSK approximation for various values of Nakagami- $m$ channel, in order to point out excellent agreement of approximated ASEP DE-QPSK values with the exact ones.

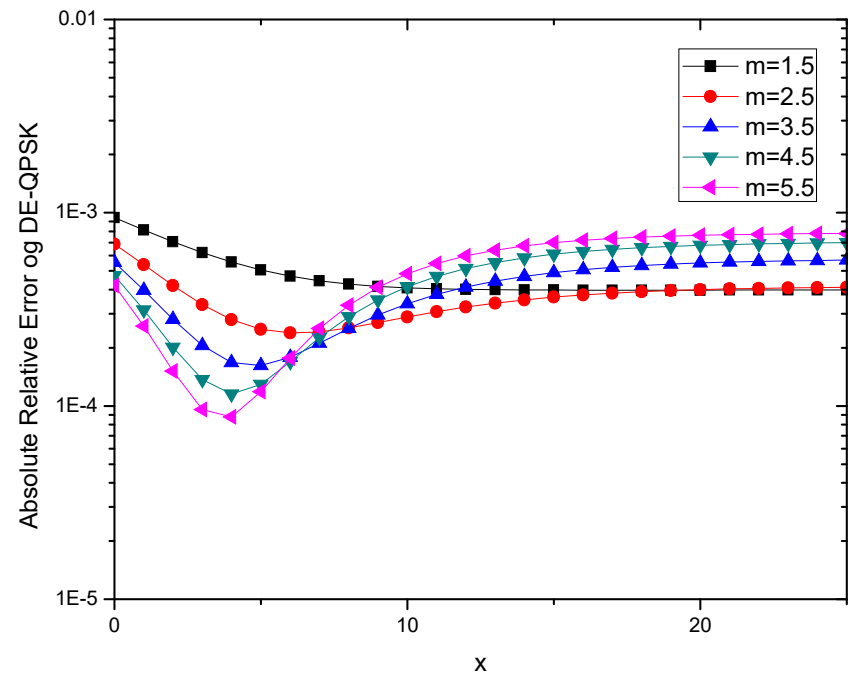

Fig. 6. Absolute relative error of proposed ABER DE-QPSK approximation.

\section{CONCLUSIONS}

In this paper, based on Mils ratio approximation of Qfunction, we have presented composite improved approximation of Q-function, and have shown that by comparing it to other known Q-function closed-form approximations, accuracy improvement has been achieved in whole range of function argument values. Further, comparisons of ASEP values over Nakagami- $m$ fading channels for various values of parameter $m$, obtained by using presented approximation of Q-function along are presented for the cases when BPSK and DE-QPSK are observed. Applicability of given approximation has been proven by showing that ASEP measures over fading channels are bounded closely by using proposed approximation.

\section{REFERENCES}

[1] L. Rubio, J. Reig, N. Cardona, "Evaluation of Nakagami fading behaviour based on measurements in urban scenarios", International Journal of Electronics and Communications (AEU), vol. 61, no. 2, pp. 135-138, 2007. [Online]. Available: http://dx.doi.org/ 10.1016/j.aeue.2006.03.004

[2] B. Selim, O. Alhussein, S. Muhaidat, G. Karagiannidis, J. Liang, "Modeling and analysis of wireless channels via the mixture of Gaussian distribution", IEEE Trans. Vehicular Technology, vol. 65, no. 10, pp. 8309-8321, 2015. [Online]. Available: http://dx.doi.org/ 10.1109/TVT.2015.2503351

[3] S. Panic, M. Stefanovic, J. Anastasov, P. Spalevic, Fading and Interference Mitigation in Wireless Communications. Florida, USA: CRC Press, 2013.

[4] P. O. Borjesson, C. E. Sundberg, "Simple approximations of the error 
function $\mathrm{Q}(\mathrm{x})$ for communications applications", IEEE Trans. Communications, vol. 27, no. 3, pp. 639-643, 1979. [Online]. Available: http://dx.doi.org/10.1109/TCOM.1979.1094433

[5] M. Chiani, D. Dardari, M. K. Simon, "New exponential bounds and approximations for the computation of error probability in fading channels", IEEE Trans. Wireless Communications, vol. 2, no. 4, pp. 840-845, 2003. [Online]. Available: http://dx.doi.org/10.1109/ TWC.2003.814350

[6] G. K. Karagiannidis, A. S. Lioumpas, "An improved approximation for the gaussian q-function", IEEE Communications Letters, vol. 11, no. 8, pp. 644-646, 2007. [Online]. Available: http://dx.doi.org/ 10.1109/LCOMM.2007.070470

[7] W. M. Jang, "A simple upper bound of the Gaussian Q-function with closed-form error bound", IEEE Communications Letters, vol. 15, no. 2, pp. 1274-1274, 2011. [Online]. Available: http://dx.doi.org/ 10.1109/LCOMM.2011.101911.111996

[8] Y. Isukapalli, B. D. Rao, "An analytically tractable approximation for the Gaussian Q-function", IEEE Communications Letters, vol. 12, no. 9, pp. 669-671, 2008. [Online]. Available: http://dx.doi.org/ 10.1109/LCOMM.2008.080815

[9] Q. Shi, Y. Karasawa, "An accurate and efficient approximation to the Gaussian Q-function and its applications in performance analysis in Nakagami-m fading", IEEE Communications Letters, vol. 15, no. 5, pp. 479-481, 2011. [Online]. Available: http://dx.doi.org/ 10.1109/LCOMM.2011.032111.102440

[10] Z.-H. Yang, Y.-M. Chu, "On approximating Mills ratio", Journal of Inequalities and Applications, 2015. [Online]. Available: $\mathrm{http}: / / \mathrm{dx}$. doi.org/10.1186/s13660-015-0792-3

[11] Marvin K. Simon, Probability Distributions Involving Gaussian Random Variables. Boston, MA: Kluwer, 2002.

[12] P. Chlumsky, J. Vodrazka, "Innovative two-path data transmission scheme proposal", Elektronika ir Elektrotechnika, vol. 20, no. 10 2014, pp. 57-61. [Online]. Available: http://dx.doi.org/10.5755/ j01.eee.20.10.5916

[13] N. Kapucu, M. Bilim, I. Develi, Y. Kabalci, "Performance of two-hop relay assisted decode-and-forward transmission under mixed fading environments", Elektronika ir Elektrotechnika, vol. 21, no. 1, 2015, pp. 60-63. [Online]. Available: http://dx.doi.org/10.5755/j01.eee. 21.1.9806

[14] P. Loskot, N. C. Beaulieu, "Prony and polynomial approximations for evaluation of the average probability of error over slow-fading channels", IEEE Trans. Vehicular Technology, vol. 58, no. 3, pp. 1269-1280, 2009. [Online]. Available: http://dx.doi.org/10.1109/ TVT.2008.926072

[15] P. C. Sofotasios, S. Freear, "Novel expressions for the Marcum and one dimensional Q-functions", Wireless Comm. Systems (ISWCS), 2010, pp. 736-740.

[16] L. M. Benitez, F. Casadevall, "Versatile, accurate, and analytically tractable approximation for the Gaussian Q-function", IEEE Trans. Communications, vol. 59, no. 4, pp. 917-922, 2011. [Online] Available: http://dx.doi.org/10.1109/TCOMM.2011.012711.100105

[17] P. Dao Ngoc, U. Nguyen Quang, H. Nguyen Xuan, R. McKay, "Evolving approximations for the Gaussian Q-function by genetic programming with semantic based crossover", Evolutionary Computation (CEC), 2012, pp. 1-6. [Online]. Available: https://doi.org/10.1109/cec.2012.6256588

[18] Y. Chen, N. C. Beaulieu, "A simple polynomial approximation to the Gaussian Q-function and its application", IEEE Communications Letters, vol. 13, no. 2, pp. 124-126, 2009. [Online]. Available: http://dx.doi.org/10.1109/LCOMM.2009.081754

[19] I. S. Gradshteyn, I. M. Ryzhik, Tables of Integrals, Series, and Products. San Diego, CA: Academic, 1994.

[20] M. K. Simon, "Single integral representations of certain integer powers of the Gaussian Q-function and their application", IEEE Communications Letters, vol. 6, no. 12, pp. 532-534, 2002. [Online]. Available: http://dx.doi.org/10.1109/LCOMM.2002.806467 